\title{
Dendritic Cells and Leishmania Infection: Adding Layers of Complexity to a Complex Disease
}

\author{
Daniel Feijó, ${ }^{1}$ Rafael Tibúrcio, ${ }^{1,2}$ Mariana Ampuero,, ${ }^{1,2}$ \\ Cláudia Brodskyn, ${ }^{1,2,3}$ and Natalia Tavares ${ }^{1}$ \\ ${ }^{1}$ Centro de Pesquisas Gonçalo Moniz (CPqGM), 40296-710 Salvador, BA, Brazil \\ ${ }^{2}$ Universidade Federal da Bahia (UFBA), 40170-115 Salvador, BA, Brazil \\ ${ }^{3}$ Instituto de Investigação em Imunologia (iii), 01246-903 São Paulo, SP, Brazil \\ Correspondence should be addressed to Daniel Feijó; danielffeijo@gmail.com and Natalia Tavares; natalia.tavares@bahia.fiocruz.br
}

Received 23 October 2015; Accepted 28 December 2015

Academic Editor: Alice O. Kamphorst

Copyright (C) 2016 Daniel Feijó et al. This is an open access article distributed under the Creative Commons Attribution License, which permits unrestricted use, distribution, and reproduction in any medium, provided the original work is properly cited.

\begin{abstract}
Leishmaniasis is a group of neglected diseases whose clinical manifestations depend on factors from the host and the pathogen. It is an important public health problem worldwide caused by the protozoan parasite from the Leishmania genus. Cutaneous Leishmaniasis (CL) is the most frequent form of this disease transmitted by the bite of an infected sandfly into the host skin. The parasites can be uptook and/or recognized by macrophages, neutrophils, and/or dendritic cells (DCs). Initially, DCs were described to play a protective role in activating the immune response against Leishmania parasites. However, several reports showed a dichotomic role of DCs in modulating the host immune response to susceptibility or resistance in CL. In this review, we discuss (1) the interactions between DCs and parasites from different species of Leishmania and (2) the crosstalk of DCs and other cells during CL infection. The complexity of these interactions profoundly affects the adaptive immune response and, consequently, the disease outcome, especially from Leishmania species of the New World.
\end{abstract}

\section{Introduction}

Leishmaniasis are a complex of vector-borne diseases caused by an intracellular protozoan parasite from Leishmania sp. (Kinetoplastida, Trypanosomatidae). Its clinical spectra depends largely on parasite species and host immune response. Although the disease has been known and studied for a long time, it is still considered as a neglected and public health problem worldwide. Such diseases affect approximately 12 million people in 88 countries, where 350 million inhabitants are exposed, mainly in remote rural areas and underserved urban areas [1]. The clinical forms range from asymptomatic infection to two main clinical syndromes: visceral leishmaniasis (VL) and cutaneous leishmaniasis (CL).

VL is a chronic infection, fatal if not treated. It is characterized by progressive fever, weight loss, splenomegaly, hepatomegaly, anemia, and spontaneous bleeding associated with marked inflammatory imbalance [2]. The hallmark of this disease is thought to be a lack of cellular immune response against the parasite and high systemic levels of IFN$g$ and IL-10 [3].

$\mathrm{CL}$ is the most frequent form of this disease. It is characterized by chronic evolution, which affects the skin and cartilaginous structures [4]. The main clinical forms of diseases associated with CL are the Localized Cutaneous Leishmaniasis (LCL), Mucocutaneous Leishmaniasis (ML), disseminated and diffuse Leishmaniasis [1].

LCL is mainly caused by the species Leishmania tropica, L. aethiopica, and L. major in the Old World. However, New World LCL is mainly caused by multiple species of both Leishmania subgenera Leishmania (L. amazonensis, L. infantum, L. mexicana, and L. venezuelensis) and Viannia subgenera ( $L$. braziliensis, L. guyanensis, L. panamensis, and L. peruviana). The incubation period lasts on average from 2 weeks to 3 months with the appearance of papules or nodules and, sometimes, is preceded or accompanied by the swelling of underlying nodes. The hallmark of this illness is the development 
of single or multiple ulcerated dermal lesions. Over time, the lesion may evolve spontaneously to healing or develop into different frames of gravity in ulceration of the lesion with its expansion [4].

Some patients (a fraction of 3\%) may develop the ML, caused by the infection with L. braziliensis and L. guyanensis. The symptoms are associated with the destruction of the nasal cavity and oropharyngeal tissues [4]. Genetic diversity of Leishmania species contributes to the difficulty of controlling the disease and to the increase in the number of cases that are resistant to conventional treatment [5]. Although both forms of CL are rarely fatal, they can cause nasty scars on the skin and severe problems in the oropharyngeal device [4].

Dendritic cells (DCs) are a family of professional antigenpresenting cells (APCs) that resides in all peripheral tissues in an immature state, capable of antigen uptake and processing. As such, they function as sentinel of the immune system. After contact with microorganisms or substances associated with infection or inflammation, DCs undergo a process of maturation and migrate to the $\mathrm{T}$ cell areas of lymphoid organs. There, they present antigens to naïve $\mathrm{T}$ cells and modulate their responses [6]. The maturation process consists of (1) increased expression of major histocompatibility complex (MHC) and costimulatory molecules, such as CD40, CD80, CD86, and CD54; (2) downregulation of antigen capture and phagocytic capacity; (3) enhanced cytokine secretion; (4) different patterns of chemokine receptor expression and chemokine production, enabling DC migration and recruitment of other cell types $[7,8]$.

DCs are able to take up antigens via different groups of receptor families, such as Fc receptors, C-type lectin receptors (CLRs), and pattern recognition receptors (PRRs), such as Toll-like receptors (TLRs) [9]. The engagement between ligand and its receptor enables DCs to recognize a wide range of microbial stimuli [10].

DCs are a heterogeneous population of cells that can be divided into 2 main categories: the plasmacytoid DCs (pDCs), experts in type I interferon synthesis, and the conventional DCs (cDCs), specialized in antigen capture, processing, and presentation for $\mathrm{T}$ cell priming. $\mathrm{pDCs}$ constitutively express MHC class II molecules and lineage markers, such as CD45RA/B220 ${ }^{+}$, Ly6C/GR-1 ${ }^{+}$, and siglec-H [11-13]. Two cDCs subsets can be distinguished based on functional specialization. $\mathrm{cDCls}$ are particularly efficient in $\mathrm{CD} 8^{+} \mathrm{T}$ cell activation and cross-presentation. cDC2s are most efficient for $\mathrm{CD}^{+} \mathrm{T}$ helper polarization, especially $\mathrm{Th} 2$ or $\mathrm{Th} 17$ [14]. In mice, $c D C 1 s$ express high levels of $\mathrm{CD} 8 \alpha$ or $\mathrm{CD} 103$ [15, 16] and cDC2s express CD11b and CD172a (also known as $\operatorname{SIRP} \alpha$ ) [17]. In humans, DCs can be subdivided into two main populations: $\mathrm{CD}_{141^{+}} \mathrm{DCs}$ (also referred to as $\mathrm{BDCA3}^{+}$) and $\mathrm{CDIc}^{+}$DCs (also known as BDCA1 ${ }^{+}$). Based on gene expression profiles and functions similarities, human CD141 ${ }^{+}$ DCs and $\mathrm{CD}_{1 c^{+}}$DCs resemble those of mouse $\mathrm{CDCl}$ s and cDC2s, respectively [18-21]. Also, monocytes can adopt a DC morphology and antigen-presenting functions in inflammatory sites, leading to their designation as monocytesderived DCs (MoDCs) $[22,23]$. In mice, MoDCs derived from Ly6 $\mathrm{C}^{\text {hi }}$ monocytes can express CD11c and MHC class II, and, similarly to macrophages, F4/80 and CD64 [23, 24].
In humans, MoDCs derived from $\mathrm{CD}_{1}{ }^{+}$monocytes and can express CD1a [24]. Langerhans cells (LCs) present DC morphology and antigen-presenting functions in the skin $[25,26]$. They constitutively express major histocompatibility complex (MHC) class II and high levels of the lectin Langerin [27]. The most current phenotypes described for each type of DC are summarized in Table 1 .

Several reports show a central role for DCs in orchestrating immune responses in leishmaniasis [28-30]. In this review, we discuss the heterogeneity of the interaction between DCs and different species of Leishmania that causes CL.

\section{Interaction of DC with Different Leishmania Species}

Infection with Leishmania parasites leads to lifelong immunity against the same subspecies, after the infection is healed. Experimental models of CL infections are largely used to study the mechanism under this lifelong immunity. Most of these studies have been carried out by inoculation of L. major, a species present in the Old World. However, experimental studies with the New World Leishmania sp., such as $L$. amazonensis and L. braziliensis, are scarce. This reinforces the importance of studies about the immune response induced by specific species of Leishmania.

2.1. Interaction of DC Subtypes with Leishmania major. Current paradigms of the involvement of $\mathrm{T}$ helper subsets in infectious diseases are based, in large part, on the results of studies about resistance and susceptibility to $L$. major in inbred mice. In murine LCL, BALB/c mice respond to infection with production of Th2-type cytokines, in particular IL-4 and IL-10. These cytokines are associated with disease progression and susceptibility to L. major. In contrast, recovery from infection of resistant mice (e.g., C57BL/6) depends on the induction of a polarized Thl-type response, resulting in macrophage activation and killing of parasites.

Early studies demonstrated that epidermal LCs phagocyte L. major in vivo and migrate to draining lymph nodes (dLNs) for presentation to antigen-specific $\mathrm{T}$ cells [31]. However, later studies showed that DCs harboring parasites in dLNs are Langerin negative and express dermal DC markers [32]. Besides, mice deficient for MHC class II exclusively in LCs (but not in dermal DCs) control L. major infection, similar to wild type animals [33]. This finding suggests that LCs are dispensable for triggering $\mathrm{T}$ cell response during Leishmania infection. Moreover, a recent study showed that LCs might even play a pathogenic role during low dose infection via the induction and expansion of regulatory $\mathrm{T}$ cells [34]. Some studies showed that dermal DCs harboring parasites migrate out of the skin and transport antigens to the dLNs $[32,35]$. Another study suggested that blood MoDCs might phagocyte parasites and transport them to the dLN, where they present parasite-derived antigen to T cells [29]. In this way, depending on the tissue and the subtype involved, DCs could have different biological response towards Leishmania interaction. 
TABLE 1: Summary of current phenotypes described for different DC subsets.

\begin{tabular}{llll}
\hline DC type & Phenotype/markers & Function & Reference \\
\hline Plasmacytoid DC (pDC) & $\begin{array}{l}\text { MHC-II, CD45RA/B220, } \\
\text { Ly6C/GR-1, Siglec-H }\end{array}$ & Type I-IFN synthesis \\
Conventional DC type 1 (cDC1) & $\begin{array}{l}\text { CD8 } \alpha, \text { CD103 (mice); } \\
\text { CD141/BDCA3 (humans) }\end{array}$ & Antigen cross-presentation, CD8 $\alpha^{+}$T cell activation & {$[15,16,18,19]$} \\
Conventional DC type 2 (cDC2) & $\begin{array}{l}\text { CD11b, CD172/SIRP } \alpha \text { (mice); } \\
\text { CD1c/BDCA1 (humans) }\end{array}$ & CD4 ${ }^{+}$T cell polarization \\
Monocyte-derived DC (MoDC) & $\begin{array}{l}\text { CD11c, MHC-II, F4/80, CD64 } \\
\text { (mice); CDla (humans) }\end{array}$ & Antigen presentation at inflammatory sites \\
Langerhans cell (LC) & MHC-II, Langerin & Antigen presentation in the skin & {$[23,24]$} \\
\hline
\end{tabular}

The production of IL-12 by APCs is critically important for the polarization of naïve $\mathrm{T}$ cells toward Thl subset and subsequent IFN- $\gamma$ production $[30,36]$. Infection of DC with L. major results in functional IL-12p70 production [37]. Interestingly, DC subsets are differentially permissive to Leishmania parasites and this differential infectivity seems to be inversely correlated with the ability of infected cells to produce IL-12p70 $[38,39]$. CD8 $\alpha^{+}$DCs are less permissive to L. major amastigotes compared to $\mathrm{CD} 8 \alpha^{-}$DCs. However, $\mathrm{CD} 8 \alpha^{+}$and $\mathrm{CD}_{103}{ }^{+}$DCs are the most powerful IL- $12 \mathrm{p} 70$ producers in response to this infection $[36,38]$. The mechanism(s) that control the induction of IL-12 from DCs and the functional differences between IL-12-producing DCs and nonproducers are still not known.

It has been speculated that different outcomes of Leishmania infection between resistant and susceptible mice may be related to differences in their DC functions, particularly in the differentiation of naïve TCD $4^{+}$into effector cells $[40,41]$. However, L. major-infected skin-derived DCs from BALB/c and $\mathrm{C} 57 \mathrm{BL} / 6$ mice upregulated costimulatory molecules and produced comparable levels of proinflammatory cytokines [30]. In further contrast, LCs from BALB/c mice upregulate IL-4 receptor expression and downregulate IL-12p40 production in response to L. major infection [42]. These findings suggest that L. major is able to inhibit Th1 immune response through altering DCs functions, depending on the cell type involved. Baldwin et al. [43] found that L. major-infected $\mathrm{BALB} / \mathrm{c}$ mice have an increased number of plasmacytoid DCs in their dLNs [43]. This was associated with increased pDC recruitment early after infection, compared to infected C57BL/6 mice.

Ashok and Acha-Orbea [44] proposed a model of infection based on DCs subtypes at the different time points after L. major infection. This model nicely explains many features and contradiction in the role of DCs subsets in cutaneous leishmaniasis: (1) dermal DCs and LCs play a role early in infection and (2) monocyte-derived dendritic cells and lymph node resident DCs are important to establish an efficient immune response at later time points [44]. However, this proposed model only focuses on DCs role in murine models based on L. major infection. It is not clear whether the differences observed in DCs from susceptible and resistant mice are relevant to the pathogenesis of the disease in humans. At present, there is still limited information on initial or late DC responses to other species of Leishmania and their contribution to prime protective or pathogenic $\mathrm{T}$ cell responses in cutaneous leishmaniasis.

2.2. Role of DCs Interaction with Other Leishmania Species. Even though cutaneous leishmaniasis is caused by almost 20 species of Leishmania, most studies about the role of DCs are focused on experimental models of 4 species: L. major, L. mexicana, L. amazonensis, and L. braziliensis.

The role of Langerhans cells (LCs) was examined in patients with different forms of cutaneous leishmaniasis (CL) caused by the New World Leishmania sp. (L. braziliensis, L. mexicana, and L. amazonensis) $[45,46]$. The analysis of LCs density among different clinical forms of CL showed a reduced LC density in L. braziliensis infection with a positive DTH response (delayed type IV hypersensibility). In comparison to nonreactive DTH from severe forms caused by L. amazonensis, an increase of LC density was observed [46]. These results indicate a species-specific negative correlation between LC density and DTH reaction among clinical forms of CL. This could lead to a suppression of T cell immune response. However, in CL caused by L. mexicana, the LCs density is similar between mild and severe clinical forms [45]. These findings indicate that L. amazonensis may use LCs to prime regulatory $\mathrm{T}$ cells, inhibiting the $\mathrm{T}$ cell responses, in a similar way to L. major infection [34].

Moreover, corroborating this clinical observation, experimental evidence confirms that early stages of L. amazonensis infection in BALB/c mice may impair multiple immune functions, leading to an antigen-specific $\mathrm{T}$ cell immune suppression [47]. Similar results were observed in murine and human DCs infected in vitro by L. amazonensis [48, 49]. However, for $L$. braziliensis murine infection, a full DC maturation process and activation were observed [50]. Together, these studies point out the specificity of strategies from different Leishmania species to modulate T cell immune response through DCs. Besides, there is a lack of information about the importance of other DC types for the development of different clinical forms caused by one species.

The dynamics of DCs migration to lymph nodes and to nonlymphoid tissues is also an important issue for the disease outcome. DCs progenitors and monocytes terminally differentiate into DCs subsets, depending on the nonlymphoid tissue they migrate, such as the skin. When activated, skin DCs upregulate CCR7 and migrate again to draining 
lymph node via afferent lymphatics in response to CCL19 and CCL21 $[14,51]$. The migration of monocyte-derived DCs to the lymph nodes is driven by CCR2 and its ligands [52]. In $\mathrm{VL}$, there is a lack of protective immune response, partially, due to an altered DC migration to the spleen and dLNs [5356]. This is also observed in CL. During L. major infection, MoDCs are preferentially recruited to the infected skin and dLN. They are important to mediate a Th1 response and to control the infection [29]. Such enhanced recruitment of DCs to dLN leads to hypertrophy of the LN, which is associated with a protective response against $L$. major [57]. On the other hand, L. mexicana infection induces limited recruitment of MoDCs and decreased LN expansion, without affecting T cell proliferation $[58,59]$. This diminished recruitment is independent of IL-10 and leads to disease progression, since treatment with neutralizing antibodies against IL-10 increases MoDCs migration and decreases parasite burden [59]. The modulation of DC recruitment to the infected skin and dLN could be used as a mechanism of immune evasion by different Leishmania sp. that causes CL.

\section{Differences in Recognition of Leishmania Parasites by DCs}

DCs express a wide variety of pattern recognition receptors (PRRs) that are important for initiating and directing subsequent adaptive immunity. The recognition of pathogen associated molecular patterns (PAMPs) can vary among species of Leishmania. de Veer et al. [60] found that MyD88 deficient $\left(\mathrm{MyD}^{-8^{-/}}\right.$) C57BL/6 mice are more susceptible to $L$. major infection, suggesting a critical role of TLR signaling in initiating anti-Leishmania immunity [60]. They further demonstrated that LPG, the most abundant surface molecule of Leishmania and a TLR2 ligand, is responsible for the generation of protective immunity against leishmaniasis. Neutralization of TLR2 and TLR4 in vivo reduced the expression of costimulatory molecules on DCs infected with L. major [61]. However, the lack of TLR2 in mice infected with $L$. braziliensis resulted in an enhanced DC activation and increased IL-12 production. As such, L. braziliensis-infected DCs from TLR2 ${ }^{-/-}$were more competent in priming naïve $\mathrm{CD}^{+} \mathrm{T}$ cells in vitro. These findings correlated with an increased IFN- $\gamma$ production in vivo and enhanced resistance to infection [62]. On the other hand, L. braziliensis-infected DCs from MyD88 ${ }^{-/-}$exhibited less activation and decreased production of interleukin-12 [62].

Furthermore, it has been shown that TLR9 signaling is crucial to the release of IL-12 and type I IFN from DCs exposed in vitro to L. major and L. braziliensis. In vivo assays with $L$. major infection also confirmed the importance of TLR9 to IL-12 production from DCs $[63,64]$. However, for L. braziliensis infection, in vivo experiments established that TLR9 $^{-/-}$mice could generate a Th1 response and activate DC, despite the diminished DC activation in vitro [65]. Together these data from TLR assays reinforce the importance to define in vitro and in vivo approaches to better characterize the modulation of DC induced by different Leishmania sp. on the immune response.
The recognition of pathogens could be optimized by the action of antibodies, a process called opsonization [66]. The uptake of L. amazonensis amastigotes by DC and LCs can be promoted by opsonization [67]. This process leads to IL10 production from these DCs, as well as the consequent priming of IL-10-producing $\mathrm{T} \mathrm{CD}^{+}$and lesion progress in mice [67]. In contrast, the uptake of opsonized L. major by murine DCs leads to cell activation, IL-12 production, and protective immunity $[68,69]$. In further contrast, L. mexicana and L. braziliensis are highly efficient in infecting DCs, even in the absence of antibodies $[62,70]$. These findings point out that the profile of cytokine production from DC is differently induced in a species-specific way, despite the same pathway recognition of Leishmania.

\section{Interaction of DC with Other Leukocytes}

Dendritic cells are the most important APCs, making a link among innate and adaptive immunity. They can have direct and diverse functions on the immune response, leading to activation as well as tolerance and anergy. In the context of CL, the functions of DCs could be modulated by the interaction with other leukocytes, such as neutrophils and NK cells.

It has been shown that genomic DNA of L. major and L. braziliensis promastigotes activate $\mathrm{cDCs}$ and $\mathrm{pDCs}$ to produce IL-12 and IFN- $\alpha / \beta$, respectively. After, they were cocultured with NK cells, leading to an increased IFN- $\gamma$ release and NK cytotoxicity [63, 64]. Certain Leishmania species (L. tropica, L. amazonensis, and L. mexicana), in their amastigotes phase, are poor inducers of IL-12 by DC. This might account for the limited NK cell response during prolonged infections in vivo [71, 72]. Hernandez Sanabria et al. [73] demonstrated that infection of L. amazonensis amastigotes triggers minimal DC activation, but the interaction with activated NK cells could partially overcome the deficiencies in DC activation in vitro [73]. The injection of activated NK cells 24 hours after infection in vivo promoted IL-12 release and increased the expression of costimulatory molecules in infected DCs (CD40, CD83, and CD80) [73]. Regarding NK cells in this context, they showed increased expression of IFN- $\gamma$ and CXCL10. Such interaction forms a positive loop, leading to the induction of a Th1 immune response to reduce parasite loads.

In a vaccination context against L. major, BALB/c depleted of NK cells and vaccinated with DCs pulsed with parasites lysates and, then, challenged with L. major showed a significant increase in footpad swelling and parasite load in the dLN [74]. In order to evaluate the mechanisms under this process, coculture of these cells was assessed. This resulted in upregulation of CD69 and IFN- $\gamma$ on NK cells as well as CD86 and MHC-II on pulsed DCs. The interaction of DC and NK cells is a good example of a positive interaction that leads to cross activation and host immune protection, either, in the context of an infection or vaccination.

Neutrophils are also an important cell type which interact with DCs. The ingestion of L. major by neutrophils in parasite-inoculated mice increased cell apoptosis. This 


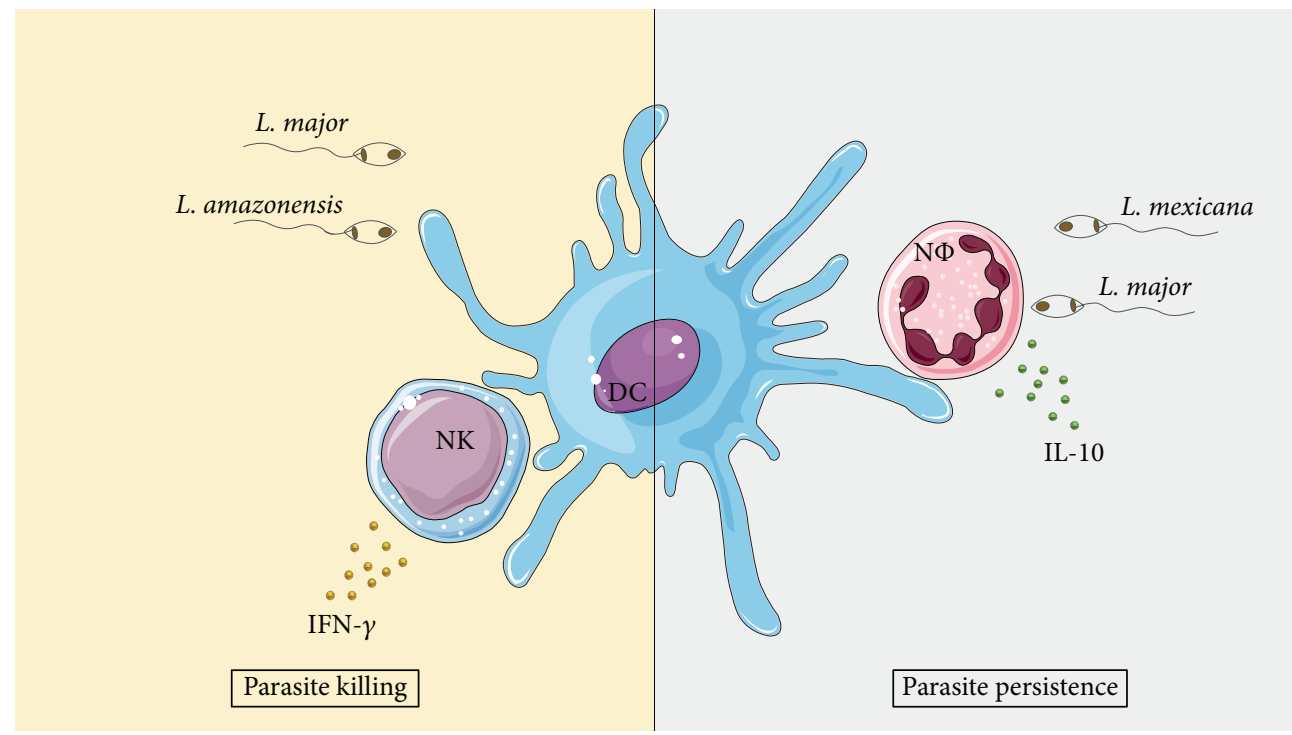

(a)

(b)

FIGURE 1: Interaction of DC with different leukocytes in the cutaneous leishmaniasis context. (a) shows the interaction among NK and infected DCs that leads to host immune protection and parasite killing through IFN- $\gamma$ production during L. amazonensis [73] or L. major [74] infection. (b) shows the outcome induced by the increased production of IL-10 after the interaction between infected neutrophils (NФ) with L. major [75] or L. mexicana [76] and DCs, leading to parasite persistence.

favored the capture of apoptotic neutrophils by DCs, preventing the activation of infected DCs in the skin [75]. In the case of L. mexicana infection, this effect was not observed, since the infection did not induce neutrophil apoptosis. Parasites sequestration by neutrophils impaired DC migration to the site of infection, through reduced CCL2, CCL3, and CCL5 release. Furthermore, the diminished DCs that migrate to the site of infection had a decreased motility and parasite uptake [76]. The interaction among DCs and neutrophils is a good example of negative regulation of the immune response, regardless of the Leishmania sp. Although the consequences of DCs and neutrophils interaction lead to immune suppression, the mechanisms of action could be diverse and Leishmania species-specific (summarized in Figure 1).

\section{Systems Biology as a Tool to Develop Vaccines against CL Based on DCs}

Recently, Matos et al. [77] showed the potential of targeting DC in vivo for induction of protective immune response against L. major in murine model of CL [77]. However, the role of DCs in CL is diverse and complex. Such role could explain some unique clinical manifestations, depending on the species of Leishmania that causes the disease. Because of that, the use of vaccines based on DCs is not yet a reality for CL. This is not only due to the complexity of the disease. Genomic and transcriptional profiles vary not only interspecifically in Leishmania parasites that causes CL [7881], but also within parasites strains isolated from patients [82]. This variability leads to a difficult task to identify a universal antigen vaccine candidate to clinical trials.
Systems Biology could be a useful tool to overcome these difficulties. Systems Biology have a holistic approach to describe complex interactions between multiple components in a biological context [83]. Using high dimensional molecular approaches, Systems Biology identifies changes caused by perturbations, such as infection or vaccination, combined with computational analysis to model and predict responses [84]. The first studies of Systems Biology about the immune response predicted that certain signatures of $\mathrm{CD}^{+} \mathrm{T}$ cells and B lymphocytes correlated with a protective immune response induced by a vaccine against Yellow Fever Virus $[85,86]$. Since then, there is an increased interest about the research of immune responses based on Systems Biology approaches. These studies lead to the identification of interactions between pathogens and hosts and factors for parasite dissemination and disease progression, as well as to the selection of promising antigens as vaccine candidates [87-89]. For instance, hub genes with unknown functions were identified from Plasmodium falciparum parasites isolated from noncerebral clinical complications of malaria. The presence of these genes correlates parasite burden and survival with complicated clinical manifestations [90]. These findings revealed the crucial roles of these genes in parasite biology and their potential as candidates for intervention strategies.

Regarding leishmaniasis, Albergante et al. [91] have developed an in silico Petri net model that simulates hepatic granuloma development during the infection in experimental visceral context. This model identified an intergranuloma diversity of the antileishmanial activity and a dominant regulatory role of IL-10 produced by infected Kupffer cells at the core of the granuloma [91]. This approach raised new insights 
into how effector mechanisms may be regulated within the granuloma and revealed a useful tool to interpret how interventions may operate. For cutaneous leishmaniasis, the analysis of DNA sequence of L. braziliensis and L. guyanensis isolated from patients with different treatment outcomes identified polymorphisms related to drug resistance [92]. This study showed that genes related to drug resistance could be used to discriminate the two species of the subgenus $L$. Viannia and also could predict treatment failure.

Those studies mentioned above demonstrate the use of System Biology as a useful tool to better understand an infection, to identify unknown pathogen cell signaling pathways, potential biomarkers of disease susceptibility, and immunological alterations that aggravates the pathology. However, the studies employing this approach are few, but they will be very promising for the development of new technologies on the leishmaniasis field.

A successful application of the System Biology approach was modeled to study the function and the role of pDC during cytopathic virus infection to identify multiscale interactions involved in the protection against the virus [93]. The results obtained from this analysis identified and predicted that (1) one infected pDC secretes sufficient type I IFN to protect up to $10^{4}$ macrophages from cytopathic viral infection; (2) pDC population in the spleen protects against virus variants which inhibit IFN production; and (3) antiviral therapy should primarily limit viral replication within peripheral organs. Together, these results demonstrate the importance of System Biology application to direct and optimize the use of different technologies based on DCs.

In this way, the application of System Biology could be a useful tool to design and develop promising vaccines candidates based on DCs pulsed with Leishmania antigens. Studies about DC signaling network based on Systems Biology approach are already published and they stand for the feasibility of this technique [94, 95]. However, the development of vaccines based on DCs through Systems Biology approach needs to be well designed to avoid undesired effects, such as the exacerbation of the CL through the increase of inflammation [96].

\section{Conclusion and Future Directions}

Given the fact that the disease pathology of CL is highly variable depending on the species of Leishmania, it is very hard to generalize specific modulatory mechanisms to all strains and in all hosts. This is important because most of the studies about the role of DCs during Leishmania infection were usually conducted with a single species of the parasite, which precludes multi-species/strain comparison. Not all Leishmania species and its interaction with DCs were studied. For instance, infection caused by L. guyanensis paradoxically induces a specific immune response via TLR3 early after infection that impairs killing of parasites [97]. A more comprehensive study would be very helpful for a better understanding about the role of these cells and the mechanisms that regulate their antigen presentation functions and also pathogen factors that could influence the antigen presentation and subsequent activation of the adaptive immune system. Besides that, the development and use of computational immunology have been constantly increasing its value. Nowadays, different in silico approaches are available for identification of potential epitopes and antigens for vaccines, since experimental methods are difficult and timeconsuming [98]. In addition, the DNA sequencing techniques became less expensive and, therefore, many parasite genome strains can be sequenced. Their predicted proteomes can be assessed considering their variability, an important feature of antigen candidates for vaccine development to one or all Leishmania species that cause CL. In this way, the use of DCs is promising for generation of potential alternatives therapies and vaccines protocols to improve the quality of life of patients infected by these protozoan parasites.

\section{Conflict of Interests}

The authors declare that they do not have a commercial association that might pose a conflict of interests.

\section{Authors' Contribution}

Cláudia Brodskyn and Natalia Tavares contributed equally to this review.

\section{Acknowledgments}

This review was supported by Conselho Nacional de Desenvolvimento Científico e Tecnológico (CNPq), Fundação de Amparo à Pesquisa do Estado da Bahia (FAPESB), and Instituto de Investigação em Imunologia-Instituto Nacional de Ciência e Tecnologia (iii-INCT), Brazil. Daniel Feijó receives fellowship from CNPq. Cláudia Brodskyn is a senior investigator at CNPq. Rafael Tibúrcio and Mariana Ampuero receive fellowships from FAPESB. The authors thank Drs. Leonardo Farias, Bruno Andrade, and Pablo Oliveira for suggestions and comments. They also thank Elaine Santos and Andrezza Souza for technical support.

\section{References}

[1] WHO/Leishmaniasis, "Leishmaniasis: Magnitude of the problem," 2014, http://www.who.int/leishmaniasis/burden/magnitude/burden_magnitude/en/index.html.

[2] S. Saha, S. Mondal, A. Banerjee, J. Ghose, S. Bhowmick, and N. Ali, "Immune responses in kala-azar," The Indian Journal of Medical Research, vol. 123, no. 3, pp. 245-266, 2006.

[3] A. Caldas, C. Favali, D. Aquino et al., "Balance of IL-10 and interferon- $\gamma$ plasma levels in human visceral leishmaniasis: implications in the pathogenesis," BMC Infectious Diseases, vol. 5, article 113, 2005.

[4] H. Goto and J. A. L. Lindoso, "Current diagnosis and treatment of cutaneous and mucocutaneous leishmaniasis," Expert Review of Anti-Infective Therapy, vol. 8, no. 4, pp. 419-433, 2010.

[5] P. J. Guerin, P. Olliaro, S. Sundar et al., "Visceral leishmaniasis: current status of control, diagnosis, and treatment, and a proposed research and development agenda," The Lancet Infectious Diseases, vol. 2, no. 8, pp. 494-501, 2002. 
[6] C. Reis e Sousa, "Activation of dendritic cells: translating innate into adaptive immunity," Current Opinion in Immunology, vol. 16, no. 1, pp. 21-25, 2004.

[7] H. Moll, "Dendritic cells and host resistance to infection," Cellular Microbiology, vol. 5, no. 8, pp. 493-500, 2003.

[8] M. Sundquist, A. Rydström, and M. J. Wick, "Immunity to Salmonella from a dendritic point of view," Cellular Microbiology, vol. 6, no. 1, pp. 1-11, 2004.

[9] T. B. H. Geijtenbeek, S. J. van Vliet, A. Engering, B. A. t Hart, and Y. van Kooyk, "Self- and nonself-recognition by C-type lectins on dendritic cells," Annual Review of Immunology, vol. 22, pp. 33-54, 2004.

[10] K. Takeda and S. Akira, "Microbial recognition by Toll-like receptors," Journal of Dermatological Science, vol. 34, no. 2, pp. 73-82, 2004.

[11] Y.-J. Liu, "IPC: professional type 1 interferon-producing cells and plasmacytoid dendritic cell precursors," Annual Review of Immunology, vol. 23, pp. 275-306, 2005.

[12] K. Shortman and Y.-J. Liu, "Mouse and human dendritic cell subtypes," Nature Reviews Immunology, vol. 2, no. 3, pp. 151-161, 2002.

[13] J. Zhang, A. Raper, N. Sugita et al., "Characterization of Siglec-H as a novel endocytic receptor expressed on murine plasmacytoid dendritic cell precursors," Blood, vol. 107, no. 9, pp. 3600-3608, 2006.

[14] M. Merad, P. Sathe, J. Helft, J. Miller, and A. Mortha, "The dendritic cell lineage: ontogeny and function of dendritic cells and their subsets in the steady state and the inflamed setting," Annual Review of Immunology, vol. 31, pp. 563-604, 2013.

[15] K. Crozat, R. Guiton, V. Contreras et al., "The XC chemokine receptor 1 is a conserved selective marker of mammalian cells homologous to mouse CD $8 \alpha^{+}$dendritic cells," The Journal of Experimental Medicine, vol. 207, no. 6, pp. 1283-1292, 2010.

[16] M. Guilliams, F. Ginhoux, C. Jakubzick et al., "Dendritic cells, monocytes and macrophages: a unified nomenclature based on ontogeny," Nature Reviews Immunology, vol. 14, no. 8, pp. 571578, 2014.

[17] S. Gurka, E. Hartung, M. Becker, and R. A. Kroczek, "Mouse conventional dendritic cells can be universally classified based on the mutually exclusive expression of XCR1 and $\operatorname{SIRP} \alpha$," Frontiers in Immunology, vol. 6, article 35, 2015.

[18] M. Haniffa, A. Shin, V. Bigley et al., "Human tissues contain CD $141^{\text {hi }}$ cross-presenting dendritic cells with functional homology to mouse $\mathrm{CD}_{103^{+}}$nonlymphoid dendritic cells," Immunity, vol. 37, no. 1, pp. 60-73, 2012.

[19] S. L. Jongbloed, A. J. Kassianos, K. J. McDonald et al., "Human $\mathrm{CD}_{141}{ }^{+}(\mathrm{BDCA}-3)^{+}$dendritic cells (DCs) represent a unique myeloid DC subset that cross-presents necrotic cell antigens," The Journal of Experimental Medicine, vol. 207, no. 6, pp. 12471260, 2010.

[20] S. H. Robbins, T. Walzer, D. Dembélé et al., "Novel insights into the relationships between dendritic cell subsets in human and mouse revealed by genome-wide expression profiling," Genome Biology, vol. 9, no. 1, article R17, 2008.

[21] A. Schlitzer, N. McGovern, P. Teo et al., "IRF4 transcription factor-dependent $\mathrm{CD}_{11 b^{+}}$dendritic cells in human and mouse control mucosal IL-17 cytokine responses," Immunity, vol. 38, no. 5, pp. 970-983, 2013.

[22] C. Cheong, I. Matos, J.-H. Choi et al., "Microbial stimulation fully differentiates monocytes to DC-SIGN/CD209 ${ }^{+}$dendritic cells for immune T cell areas," Cell, vol. 143, no. 3, pp. 416-429, 2010.
[23] C. Langlet, S. Tamoutounour, S. Henri et al., "CD64 expression distinguishes monocyte-derived and conventional dendritic cells and reveals their distinct role during intramuscular immunization," Journal of Immunology, vol. 188, no. 4, pp. 1751-1760, 2012.

[24] S. Tamoutounour, M. Guilliams, F. M. Sanchis et al., "Origins and functional specialization of macrophages and of conventional and monocyte-derived dendritic cells in mouse skin," Immunity, vol. 39, no. 5, pp. 925-938, 2013.

[25] M. Greter, I. Lelios, P. Pelczar et al., "Stroma-derived interleukin-34 controls the development and maintenance of langerhans cells and the maintenance of microglia," Immunity, vol. 37, no. 6, pp. 1050-1060, 2012.

[26] J. C. Miller, B. D. Brown, T. Shay et al., "Deciphering the transcriptional network of the dendritic cell lineage," Nature Immunology, vol. 13, no. 9, pp. 888-899, 2012.

[27] M. Merad and M. G. Manz, "Dendritic cell homeostasis," Blood, vol. 113, no. 15, pp. 3418-3427, 2009.

[28] P. M. A. Gorak, C. R. Engwerda, and P. M. Kaye, "Dendritic cells, but not macrophages, produce IL-12 immediately following Leishmania donovani infection," European Journal of Immunology, vol. 28, no. 2, pp. 687-695, 1998.

[29] B. León, M. López-Bravo, and C. Ardavín, "Monocyte-derived dendritic cells formed at the infection site control the induction of protective T helper 1 responses against Leishmania," Immunity, vol. 26, no. 4, pp. 519-531, 2007.

[30] E. von Stebut, Y. Belkaid, T. Jakob, D. L. Sacks, and M. C. Udey, "Uptake of Leishmania major amastigotes results in activation and interleukin 12 release from murine skin-derived dendritic cells: implications for the initiation of anti-Leishmania immunity," The Journal of Experimental Medicine, vol. 188, no. 8, pp. 1547-1552, 1998.

[31] H. Moll, H. Fuchs, C. Blank, and M. Rollinghoff, "Langerhans cells transport Leishmania major from the infected skin to the draining lymph node for presentation to antigen-specific $\mathrm{T}$ cells," European Journal of Immunology, vol. 23, no. 7, pp. 15951601, 1993.

[32] U. Ritter, A. Meißner, C. Scheidig, and H. Körner, "CD8 $\alpha$ - and Langerin-negative dendritic cells, but not Langerhans cells, act as principal antigen-presenting cells in leishmaniasis," European Journal of Immunology, vol. 34, no. 6, pp. 1542-1550, 2004.

[33] M. P. Lemos, F. Esquivel, P. Scott, and T. M. Laufer, "MHC class II expression restricted to $\mathrm{CD} 8 \alpha^{+}$and $\mathrm{CD}_{11 \mathrm{~b}}{ }^{+}$dendritic cells is sufficient for control of Leishmania major," Journal of Experimental Medicine, vol. 199, no. 5, pp. 725-730, 2004.

[34] K. Kautz-Neu, M. Noordegraaf, S. Dinges et al., "Langerhans cells are negative regulators of the anti-Leishmania response," The Journal of Experimental Medicine, vol. 208, no. 5, pp. 885891, 2011.

[35] L. G. Ng, A. Hsu, M. A. Mandell et al., "Migratory dermal dendritic cells act as rapid sensors of protozoan parasites," PLoS Pathogens, vol. 4, no. 11, Article ID e1000222, 2008.

[36] M. Martínez-López, S. Iborra, R. Conde-Garrosa, and D. Sancho, "Batf3-dependent $\mathrm{CD}_{103}{ }^{+}$dendritic cells are major producers of IL-12 that drive local Th1 immunity against Leishmania major infection in mice," European Journal of Immunology, vol. 45, no. 1, pp. 119-129, 2015.

[37] M. A. Marovich, M. A. McDowell, E. K. Thomas, and T. B. Nutman, "IL-12p70 production by Leishmania major-harboring human dendritic cells is a CD40/CD40 ligand-dependent process," Journal of Immunology, vol. 164, no. 11, pp. 5858-5865, 2000 . 
[38] S. Henri, J. Curtis, H. Hochrein, D. Vremec, K. Shortman, and E. Handman, "Hierarchy of susceptibility of dendritic cell subsets to infection by Leishmania major: inverse relationship to interleukin-12 production," Infection and Immunity, vol. 70, no. 7, pp. 3874-3880, 2002.

[39] M. Akbari, K. Honma, D. Kimura et al., "IRF4 in dendritic cells inhibits IL-12 production and controls TH1 immune responses against leishmania major," Journal of Immunology, vol. 192, no. 5, pp. 2271-2279, 2014.

[40] K. Suzue, S. Kobayashi, T. Takeuchi, M. Suzuki, and S. Koyasu, "Critical role of dendritic cells in determining the Th1/Th2 balance upon Leishmania major infection," International Immunology, vol. 20, no. 3, pp. 337-343, 2008.

[41] M. J. Girard-Madoux, K. Kautz-Neu, B. Lorenz, J. L. OberBlöbaum, E. von Stebut, and B. E. Clausen, "IL-10 signaling in dendritic cells attenuates anti-Leishmania major immunity without affecting protective memory responses," Journal of Investigative Dermatology, vol. 135, no. 11, pp. 2890-2894, 2015.

[42] H. Moll, A. Scharner, and E. Kämpgen, "Increased interleukin 4 (IL-4) receptor expression and IL-4-induced decrease in IL-12 production by langerhans cells infected with Leishmania major," Infection and Immunity, vol. 70, no. 3, pp. 1627-1630, 2002.

[43] T. M. Baldwin, C. Elso, J. Curtis, L. Buckingham, and E. Handman, "The site of Leishmania major infection determines disease severity and immune responses," Infection and Immunity, vol. 71, no. 12, pp. 6830-6834, 2003.

[44] D. Ashok and H. Acha-Orbea, "Timing is everything: dendritic cell subsets in murine Leishmania infection," Trends in Parasitology, vol. 30, no. 10, pp. 499-507, 2014.

[45] U. Ritter, H. Moll, T. Laskay et al., "Differential expression of chemokines in patients with localized and diffuse cutaneous American leishmaniasis," The Journal of Infectious Diseases, vol. 173, no. 3, pp. 699-709, 1996.

[46] M. B. Xavier, F. T. Silveira, S. Demachki, M. M. R. Ferreira, and J. L. M. do Nascimento, "American tegumentary leishmaniasis: a quantitative analysis of Langerhans cells presents important differences between L. (L.) amazonensis and Viannia subgenus," Acta Tropica, vol. 95, no. 1, pp. 67-73, 2005.

[47] J. Ji, J. Sun, and L. Soong, "Impaired expression of inflammatory cytokines and chemokines at early stages of infection with Leishmania amazonensis," Infection and Immunity, vol. 71, no. 8, pp. 4278-4288, 2003.

[48] C. Favali, N. Tavares, J. Clarêncio, A. Barral, M. Barral-Netto, and C. Brodskyn, "Leishmania amazonensis infection impairs differentiation and function of human dendritic cells," Journal of Leukocyte Biology, vol. 82, no. 6, pp. 1401-1406, 2007.

[49] L. Soong, "Modulation of dendritic cell function by leishmania parasites," Journal of Immunology, vol. 180, no. 7, pp. 4355-4360, 2008.

[50] A. K. Carvalho, F. T. Silveira, L. F. D. Passero, C. M. C. Gomes, C. E. P. Corbett, and M. D. Laurenti, "Leishmania (V.) braziliensis and L. (L.) amazonensis promote differential expression of dendritic cells and cellular immune response in murine model," Parasite Immunology, vol. 34, no. 8-9, pp. 395-403, 2012.

[51] L. Ohl, M. Mohaupt, N. Czeloth et al., "CCR7 governs skin dendritic cell migration under inflammatory and steady-state conditions," Immunity, vol. 21, no. 2, pp. 279-288, 2004.

[52] F. Geissmann, S. Gordon, D. A. Hume, A. M. Mowat, and G. J. Randolph, "Unravelling mononuclear phagocyte heterogeneity," Nature Reviews Immunology, vol. 10, no. 6, pp. 453-460, 2010.
[53] M. K. Ibrahim, J. L. Barnes, E. Y. Osorio et al., "Deficiency of lymph node-resident dendritic cells (DCs) and dysregulation of DC chemoattractants in a malnourished mouse model of Leishmania donovani infection," Infection and Immunity, vol. 82, no. 8, pp. 3098-3112, 2014.

[54] M. Ato, A. Maroof, S. Zubairi, H. Nakano, T. Kakiuchi, and P. M. Kaye, "Loss of dendritic cell migration and impaired resistance to Leishmania donovani infection in mice deficient in CCL19 and CCL21," The Journal of Immunology, vol. 176, no. 9, pp. 5486-5493, 2006.

[55] M. Ato, S. Stäger, C. R. Engwerda, and P. M. Kaye, "Defective CCR7 expression on dendritic cells contributes to the development of visceral leishmaniasis," Nature Immunology, vol. 3, no. 12, pp. 1185-1191, 2002.

[56] A. C. Stanley, J. E. Dalton, S. H. Rossotti et al., "VCAM-1 and VLA-4 modulate dendritic cell IL-12p40 production in experimental visceral leishmaniasis," PLoS Pathogens, vol. 4, no. 9, Article ID e1000158, 2008.

[57] L. P. Carvalho, P. M. Petritus, A. L. Trochtenberg et al., "Lymph node hypertrophy following Leishmania major infection is dependent on TLR9," The Journal of Immunology, vol. 188, no. 3, pp. 1394-1401, 2012.

[58] A. C. Hsu and P. Scott, "Leishmania mexicana infection induces impaired lymph node expansion and Th1 cell differentiation despite normal T cell proliferation," The Journal of Immunology, vol. 179, no. 12, pp. 8200-8207, 2007.

[59] P. M. Petritus, D. Manzoni-de-Almeida, C. Gimblet, C. Gonzalez Lombana, and P. Scott, "Leishmania mexicana mexicana induces limited recruitment and activation of monocytes and monocyte-derived dendritic cells early during infection," PLoS Neglected Tropical Diseases, vol. 6, no. 10, Article ID e1858, 2012.

[60] M. J. de Veer, J. M. Curtis, T. M. Baldwin et al., "MyD88 is essential for clearance of Leishmania major: possible role for lipophosphoglycan and Toll-like receptor 2 signaling," European Journal of Immunology, vol. 33, no. 10, pp. 2822-2831, 2003.

[61] M. Komai-Koma, D. Li, E. Wang, D. Vaughan, and D. Xu, "AntiToll-like receptor 2 and 4 antibodies suppress inflammatory response in mice," Immunology, vol. 143, no. 3, pp. 354-362, 2014.

[62] D. A. Vargas-Inchaustegui, W. Tai, L. Xin, A. E. Hogg, D. B. Corry, and L. Soong, "Distinct roles for MyD88 and toll-like receptor 2 during Leishmania braziliensis infection in mice," Infection and Immunity, vol. 77, no. 7, pp. 2948-2956, 2009.

[63] J. Liese, U. Schleicher, and C. Bogdan, "TLR9 signaling is essential for the innate NK cell response in murine cutaneous leishmaniasis," European Journal of Immunology, vol. 37, no. 12, pp. 3424-3434, 2007.

[64] U. Schleicher, J. Liese, I. Knippertz et al., "NK cell activation in visceral leishmaniasis requires TLR9, myeloid DCs, and IL12 , but is independent of plasmacytoid DCs," The Journal of Experimental Medicine, vol. 204, no. 4, pp. 893-906, 2007.

[65] T. Weinkopff, A. Mariotto, G. Simon et al., "Role of toll-like receptor 9 signaling in experimental Leishmania braziliensis infection," Infection and Immunity, vol. 81, no. 5, pp. 1575-1584, 2013.

[66] L. T. Vogelpoel, D. L. Baeten, E. C. de Jong, and J. den Dunnen, "Control of cytokine production by human fc gamma receptors: implications for pathogen defense and autoimmunity," Frontiers in Immunology, vol. 6, article 79, 2015.

[67] N. Wanasen, L. Xin, and L. Soong, "Pathogenic role of B cells and antibodies in murine Leishmania amazonensis infection," 
International Journal for Parasitology, vol. 38, no. 3-4, pp. 417429, 2008.

[68] A. P. Nigg, S. Zahn, D. Rückerl et al., "Dendritic cell-derived IL-12p40 homodimer contributes to susceptibility in cutaneous leishmaniasis in BALB/c mice," The Journal of Immunology, vol. 178, no. 11, pp. 7251-7258, 2007.

[69] F. Woelbing, S. L. Kostka, K. Moelle et al., "Uptake of Leishmania major by dendritic cells is mediated by $\mathrm{Fc} \gamma$ receptors and facilitates acquisition of protective immunity," The Journal of Experimental Medicine, vol. 203, no. 1, pp. 177-188, 2006.

[70] E. Prina, S. Z. Abdi, M. Lebastard, E. Perret, N. Winter, and J.C. Antoine, "Dendritic cells as host cells for the promastigote and amastigote stages of Leishmania amazonensis: the role of opsonins in parasite uptake and dendritic cell maturation," Journal of Cell Science, vol. 117, no. 2, pp. 315-325, 2004.

[71] C. L. Bennett, A. Misslitz, L. Colledge, T. Aebischer, and C. C. Blackburn, "Silent infection of bone marrow-derived dendritic cells by Leishmania mexicana amastigotes," European Journal of Immunology, vol. 31, no. 3, pp. 876-883, 2001.

[72] M. A. McDowell, M. Marovich, R. Lira, M. Braun, and D. Sacks, "Leishmania priming of human dendritic cells for CD40 ligand-induced interleukin-12p70 secretion is strain and species dependent," Infection and Immunity, vol. 70, no. 8, pp. 39944001, 2002.

[73] M. X. Hernandez Sanabria, D. A. Vargas-Inchaustegui, L. Xin, and L. Soong, "Role of natural killer cells in modulating dendritic cell responses to Leishmania amazonensis infection," Infection and Immunity, vol. 76, no. 11, pp. 5100-5109, 2008.

[74] K. A. Remer, B. Roeger, C. Hambrecht, and H. Moll, "Natural killer cells support the induction of protective immunity during dendritic cell-mediated vaccination against Leishmania major," Immunology, vol. 131, no. 4, pp. 570-582, 2010.

[75] F. L. Ribeiro-Gomes, N. C. Peters, A. Debrabant, and D. L. Sacks, "Efficient capture of infected neutrophils by dendritic cells in the skin inhibits the early anti-leishmania response," PLoS Pathogens, vol. 8, no. 2, Article ID e1002536, 2012.

[76] B. P. Hurrell, S. Schuster, E. Grün et al., "Rapid sequestration of Leishmania mexicana by neutrophils contributes to the development of chronic lesion," PLoS Pathogens, vol. 11, no. 5, Article ID e1004929, 2015.

[77] I. Matos, O. Mizenina, A. Lubkin, R. M. Steinman, and J. Idoyaga, "Targeting antigens to dendritic cells in vivo induces protective immunity," PLoS ONE, vol. 8, no. 6, Article ID e67453, 2013.

[78] L. A. Dillon, K. Okrah, V. K. Hughitt et al., "Transcriptomic profiling of gene expression and RNA processing during Leishmania major differentiation," Nucleic Acids Research, vol. 43, no. 14, pp. 6799-6813, 2015.

[79] M. Fiebig, S. Kelly, E. Gluenz, and P. J. Myler, "Comparative life cycle transcriptomics revises Leishmania mexicana genome annotation and links a chromosome duplication with parasitism of vertebrates," PLoS Pathogens, vol. 11, no. 10, Article ID e1005186, 2015.

[80] C. S. Peacock, K. Seeger, D. Harris et al., "Comparative genomic analysis of three Leishmania species that cause diverse human disease," Nature Genetics, vol. 39, no. 7, pp. 839-847, 2007.

[81] D. A. Tschoeke, G. L. Nunes, R. Jardim et al., "The comparative genomics and phylogenomics of Leishmania amazonensis parasite," Evolutionary Bioinformatics, vol. 10, pp. 131-153, 2014.

[82] E. V. Alves-Ferreira, J. S. Toledo, A. H. De Oliveira et al., "Differential gene expression and infection profiles of cutaneous and mucosal Leishmania braziliensis isolates from the same patient," PLoS Neglected Tropical Diseases, vol. 9, no. 9, Article ID e0004018, 2015.

[83] L. H. Hartwell, J. J. Hopfield, S. Leibler, and A. W. Murray, "From molecular to modular cell biology," Nature, vol. 402, no. 6761, supplement, pp. C47-C52, 1999.

[84] H. Kitano, "Systems biology: a brief overview," Science, vol. 295, no. 5560, pp. 1662-1664, 2002.

[85] D. Gaucher, R. Therrien, N. Kettaf et al., "Yellow fever vaccine induces integrated multilineage and polyfunctional immune responses," The Journal of Experimental Medicine, vol. 205, no. 13, pp. 3119-3131, 2008.

[86] T. D. Querec, R. S. Akondy, E. K. Lee et al., "Systems biology approach predicts immunogenicity of the yellow fever vaccine in humans," Nature Immunology, vol. 10, no. 1, pp. 116-125, 2009.

[87] C. J. Blohmke, D. O'Connor, and A. J. Pollard, "The use of systems biology and immunological big data to guide vaccine development," Genome Medicine, vol. 7, no. 1, article 114, 2015.

[88] J. B. Gutierrez, M. R. Galinski, S. Cantrell, and E. O. Voit, "From within host dynamics to the epidemiology of infectious disease: scientific overview and challenges," Mathematical Biosciences B, vol. 270, pp. 143-155, 2015.

[89] D. E. Zak and A. Aderem, "Systems integration of innate and adaptive immunity," Vaccine, vol. 33, no. 40, pp. 5241-5248, 2015.

[90] A. K. Subudhi, P. A. Boopathi, I. Pandey et al., "Disease specific modules and hub genes for intervention strategies: a coexpression network based approach for Plasmodium falciparum clinical isolates," Infection, Genetics and Evolution, vol. 35, pp. 96-108, 2015.

[91] L. Albergante, J. Timmis, L. Beattie, and P. M. Kaye, "A Petri net model of granulomatous inflammation: implications for IL10 mediated control of Leishmania donovani infection," PLoS Computational Biology, vol. 9, no. 11, Article ID e1003334, 2013.

[92] D. C. Torres, M. Ribeiro-Alves, G. A. S. Romero, A. M. R. Dávila, and E. Cupolillo, "Assessment of drug resistance related genes as candidate markers for treatment outcome prediction of cutaneous leishmaniasis in Brazil," Acta Tropica, vol. 126, no. 2, pp. 132-141, 2013.

[93] G. Bocharov, R. Züst, L. Cervantes-Barragan et al., "A systems immunology approach to plasmacytoid dendritic cell function in cytopathic virus infections," PLoS Pathogens, vol. 6, no. 7, Article ID e1001017, pp. 1-15, 2010.

[94] D. Cavalieri, D. Rivero, L. Beltrame et al., "DC-ATLAS: a systems biology resource to dissect receptor specific signal transduction in dendritic cells," Immunome Research, vol. 6, no. 1, article 10, 2010.

[95] S. Patil, H. Pincas, J. Seto, G. Nudelman, I. Nudelman, and S. C. Sealfon, "Signaling network of dendritic cells in response to pathogens: a community-input supported knowledgebase," BMC Systems Biology, vol. 4, article 137, 2010.

[96] P. Tsagozis, E. Karagouni, and E. Dotsika, "Dendritic cells pulsed with peptides of gp63 induce differential protection against experimental cutaneous leishmaniasis," International Journal of Immunopathology and Pharmacology, vol. 17, no. 3, pp. 343-352, 2004.

[97] A. Ives, C. Ronet, F. Prevel et al., "Leishmania RNA virus controls the severity of mucocutaneous leishmaniasis," Science, vol. 331, no. 6018, pp. 775-778, 2011.

[98] D. R. Flower, I. K. Macdonald, K. Ramakrishnan, M. N. Davies, and I. A. Doytchinova, "Computer aided selection of candidate vaccine antigens," Immunome Research, vol. 6, supplement 2, article S1, 2010. 


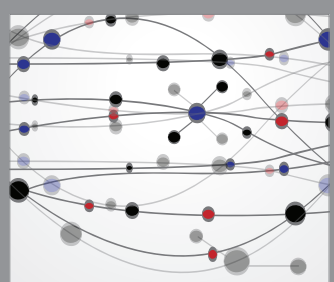

The Scientific World Journal
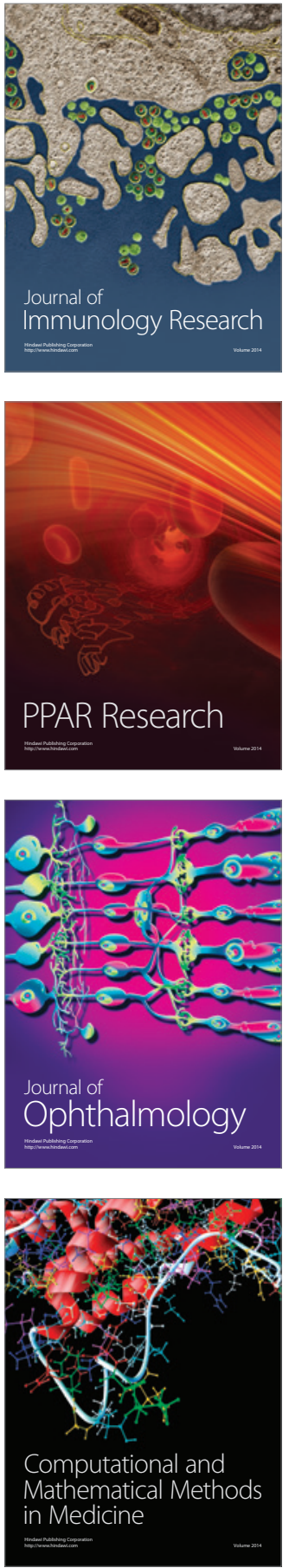

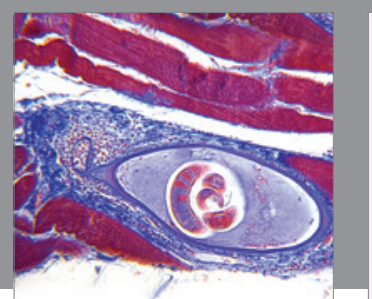

Gastroenterology Research and Practice

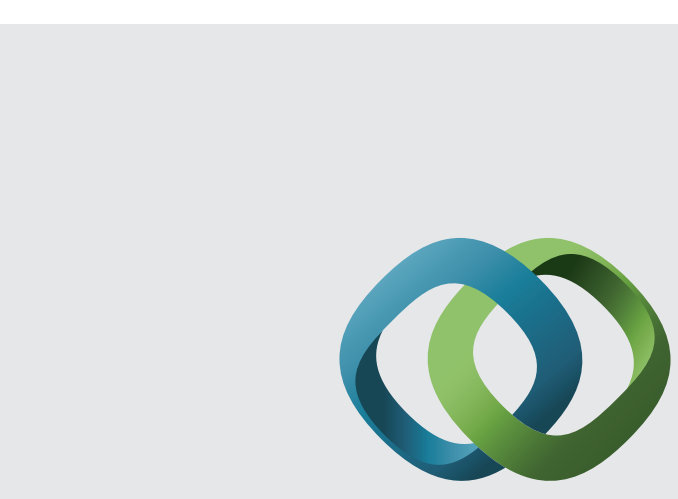

\section{Hindawi}

Submit your manuscripts at

http://www.hindawi.com
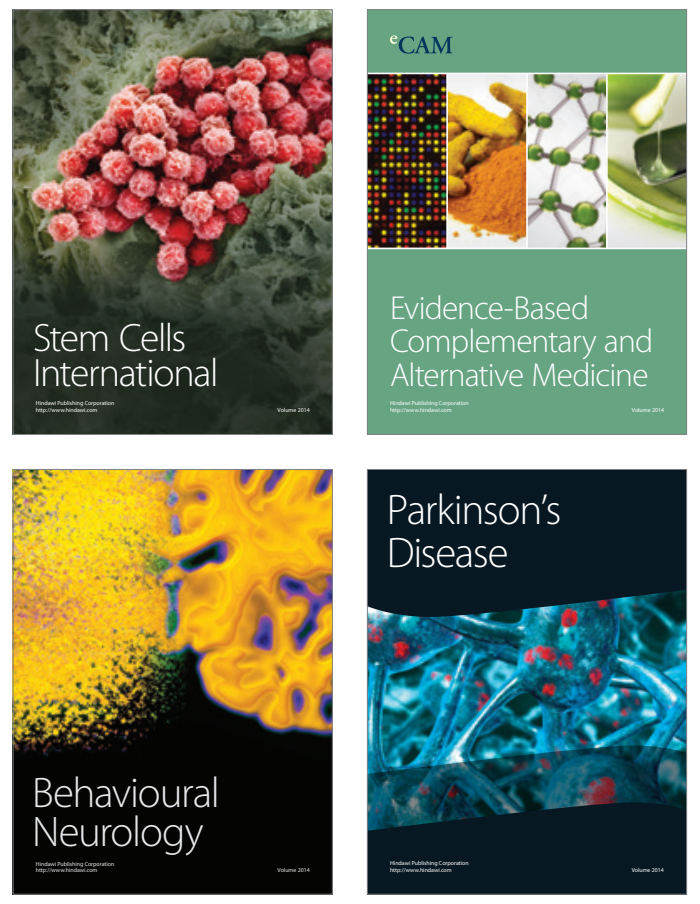
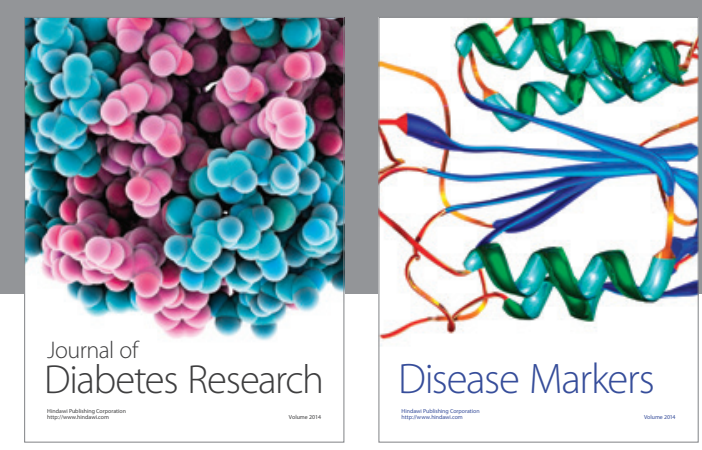

Disease Markers
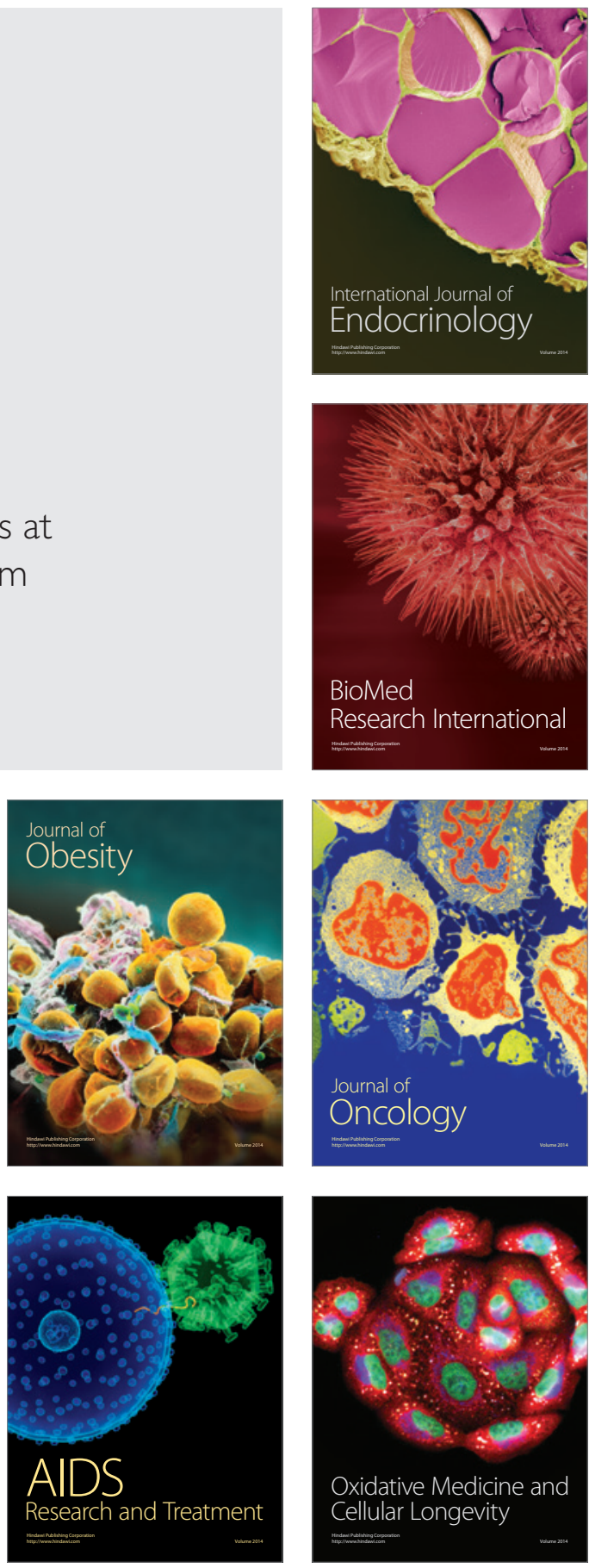